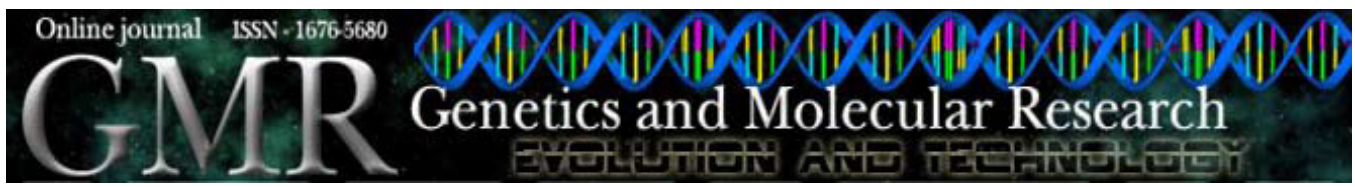

\title{
Lack of association of immune-response-gene polymorphisms with susceptibility to sarcoidosis in Slovenian patients
}

\author{
A. Maver ${ }^{1}$, I. Medica ${ }^{1}$, B. Salobir ${ }^{2}$, M. Tercelj ${ }^{2}$ and B. Peterlin ${ }^{1}$ \\ ${ }^{1}$ Department of Obstetrics and Gynecology, Institute of Medical Genetics, \\ University Medical Centre, Ljubljana, Slovenia \\ ${ }^{2}$ Department of Pulmonary Diseases and Allergy, \\ University Medical Centre, Ljubljana, Slovenia
}

Corresponding author: B. Peterlin

E-mail: borut.peterlin@guest.arnes.si

Genet. Mol. Res. 9 (1): 58-68 (2010)

Received October 13, 2009

Accepted December 20, 2009

Published January 12, 2010

\begin{abstract}
Sarcoidosis is a chronic inflammatory disease, characterized by granulomatous inflammation, prominently involving the respiratory system. The etiology of this disease has not yet been elucidated and the contribution of genetic is not yet completely understood. We searched for novel candidate genes, utilizing a system biology approach, based on data from published transcriptional, proteomic and linkage studies of sarcoidosis. The search revealed several new potential candidate genes involved in the pathogenesis of inflammatory lung diseases: $25-(\mathrm{OH})-$ vitamin $\mathrm{D}_{3}-1 \alpha$-hydroxylase $(C Y P 27 B 1)$, endothelin-1 (EDN1) and glutathione S-transferase Pi (GSTP1). Variants of selected polymorphisms: -1260/ C >A in CYP27B1, Lys198Asn in EDN1, and Ile105Val in GSTP1, were examined to determine if they confer susceptibility to sarcoidosis, based on an analysis of 180 Slovenian patients in comparison with 283 healthy controls. Polymerase chain reactions using allele-specific oligonucleotides were performed. This disease was not significantly associated with genotypes $\mathrm{CC}$ at $-1260 / \mathrm{C}>\mathrm{A}$ polymorphism in CYP27B1 $(\mathrm{P}=0.68$, odds ratio $(\mathrm{OR})=1.10,95 \%$ confidence interval $(\mathrm{CI})=0.75-1.61), \mathrm{GG}$ genotype at Lys198Asn polymorphism in EDN1 $(\mathrm{P}=1.00$, OR $=0.97$, $95 \% \mathrm{CI}=0.65-1.44)$ and AA genotypes at Ile105Val polymorphism in
\end{abstract}


GSTP1 $(\mathrm{P}=0.53, \mathrm{OR}=0.87,95 \% \mathrm{CI}=0.60-1.27)$. There was no association of polymorphisms in any of the genes with sarcoidosis.

Key words: Association study; CYP27B1; Genetic polymorphisms; EDN1; GSTP1; Sarcoidosis

\section{INTRODUCTION}

Sarcoidosis is a chronic multisystemic inflammatory disorder characterized by granulomatous inflammation that primarily affects the respiratory system but may involve other organ systems. The immunologic phenomena that characterize sarcoid inflammation involve diffuse infiltration of parenchymal organs with CD4+ T lymphocytes of the $T_{H} 1$ type, formation of granulomas and distortion of normal organ micro-architecture, which can eventually disturb normal function of the involved organ (Baughman et al., 2003).

The etiological basis of sarcoidosis has not yet been elucidated. Possible hypotheses implicate various infectious, environmental and genetic factors, but none has been consistently associated with this disease. Sarcoidosis develops as a result of the interplay between various environmental agents acting on a genetically susceptible host. This genetic susceptibility has been the subject of intensive research, but few studies have reported significant associations, none of which can completely explain the genetic background of sarcoidosis. Therefore, there is still a need to investigate genetic factors contributing to the development of this disease (Hunninghake et al., 1999).

Various approaches can be utilized to explore possible genetic associations with diseases. The candidate gene approach is commonly used to search for potential disease-associated candidate genes. It involves searching for plausible genes and later evaluating the chosen genes in an actual study to confirm whether the association is significant or not. The first part, the search for possible gene candidates, is often done at a symbolic level, based on the investigators knowledge and intuition. With the introduction of high-throughput technologies, detailed information about the genomic, transcriptomic and proteomic profile of sarcoidosis has become available (Schurmann et al., 2001; Rutherford et al., 2001, 2004; Sabounchi-Schutt et al., 2003, 2004). Relating this knowledge to the candidate-gene search process could significantly increase the possibility of finding significant gene-disease associations. Using this approach, we obtained a list of potential gene candidates for sarcoidosis. Further literature search revealed that several of these candidates have recently been found to play a role in the immune response regulation but have not yet been implicated in genetic association studies of sarcoidosis. We therefore chose to study functional polymorphisms in the 25-(OH)-vitamin $\mathrm{D}_{3}$ 1 $\alpha$-hydroxylase (CYP27B1), endothelin-1 (EDN1) and glutathione S-transferase Pi (GSTP1) genes in sarcoidosis patients. $C Y P 27 B 1$ codes for $25-(\mathrm{OH})$-vitamin $\mathrm{D}_{3}-1 \alpha$-hydroxylase, a member of the cytochrome P450 superfamily of enzymes, which is responsible for the synthesis of an active form of vitamin $\mathrm{D}$, important in the regulation of body calcium homeostasis (Kong et al., 1999). Sarcoidosis is characterized by hypercalcemia in about $2-10 \%$ of patients and about three times more often by hypercalciuria, which is related to aberrant production of 1,25-(OH) $)_{2}$-vitamin $\mathrm{D}_{3}$ (calcitriol) (Hunninghake et al., 1999). Calcitriol has also been related to immune-response regulation (Cantorna et al., 2004). A variant of a polymorphism in the promoter region of CYP27B1 (-1260/ C >A, rs3087459) has been associated with various diseases of the immune system (Lopez et al., 2004). 
Endothelin-1 peptide (ET-1) has consistently been found to be elevated in patients with sarcoidosis (Letizia et al., 2001; Reichenberger et al., 2001). It is generally considered to be a powerful vasoconstricting substance, but recent reports show that its levels are increased in several inflammatory and fibrotic lung diseases as well (Reichenberger et al., 2001). ET-1 is coded by EDN1 and polymorphisms in the gene region, of which Lys198Asn (K198N, rs5370) in exon 5 has previously been associated with hypertension (Jin et al., 2003).

GSTP1 codes for an enzyme of the glutathione S-transferase class, involved in detoxification reactions. The GSTP1 enzyme has been previously examined for its roles in the etiology of respiratory diseases, in the regulating of the extent of oxidative damage of tissues and in inactivation of reactive oxygen species (ROS) (Montano et al., 2004). ROS have also been implicated in the regulation of inflammatory processes, and their role in various inflammatory disorders has been established (Rahman, 2003). We chose to study the GSTP1 gene, as sarcoidosis is characterized by increased oxidative damage and increased levels of GSTP1 enzyme (Sabounchi-Schutt et al., 2004). A single nucleotide polymorphism (SNP) in the GSTP1 gene, resulting from a single base change at codon 105 (Ile105Val, rs1695), was selected for our analysis.

\section{MATERIAL AND METHODS}

\section{Candidate gene search}

Initially, we searched the Pubmed database (http://www.pubmed.com/) for existing association studies of sarcoidosis. Genes, that were previously studied for association with sarcoidosis, were excluded from further analyses. We then searched Pubmed for existing linkage, transcriptomic and proteomic studies of sarcoidosis; the results of these studies were entered into our database of candidate genes. Additional information regarding gene function was found using the GeneOntology database (http://www.geneontology.org/) and also entered this into our database. We also used literature discovery support algorithms, such as BITOLA (http://www.mf.uni-lj.si/bitola/), in order to find new potentially meaningful relations that already exist in the published literature. With this information, the linkage, transcriptomic, proteomic, functional, and pathophysiological data were considered, and new candidate genes with the most interesting aspects were advanced to candidates in an association study. Microsoft Access $^{\circledR}$ was used as a database tool for collection of information on candidate genes.

\section{Patients and controls}

Sarcoidosis patients were recruited from an ongoing sarcoidosis registry started in 2000 at the Ljubljana University Medical Centre, Department of Pulmology and Allergic Diseases. Diagnosis of sarcoidosis was based on the clinical picture, radiographic presentation, bronchoalveolar lavage, and biopsy specimens from the lung, skin or lymph nodes after other granulomatous diseases were excluded. A hundred and eighty patients were included in our study. The patients ( 75 males and 105 females) had a mean age of 42.5 years at the time of diagnosis, with a standard deviation of 13.3. Pulmonary lymph nodes were affected in 150, and lung interstitium in 138 patients. According to the classification system based on established guidelines (Hunninghake et al., 1999), 28 patients were in stage I, 105 in stage II, 29 in 
stage III, and 5 in stage IV of the disease at first presentation. Different types of skin involvement were found in 48 patients, Löfgren's syndrome was found in 29 patients, 28 patients had arthralgias, 23 patients had extrapulmonary lymph node involvement, and 6 patients had salivary glands involvement; in 15 patients other organs (liver, spleen, kidney, heart), and in 14 patients different types of neural involvement were found. In 47 patients the disease was still active after two years of follow-up; thus, they were diagnosed with chronic sarcoidosis, whereas 101 patients who recovered within two years after diagnosis were diagnosed with acute sarcoidosis. The remaining 32 patients were diagnosed within the previous two years, and therefore had not been classified yet. The control group consisted of 283 healthy randomly recruited blood donors, 117 males and 166 females, with a mean age of 39.2 years and a standard deviation of 11.0 years. All patient and control subjects were Slovenian, not related to each other. All the study subjects participated after they had given their full informed consent. The study was approved by the National Medical Ethics Committee.

\section{Genotyping at three polymorphisms}

After DNA isolation from blood leukocytes by standard procedures, genotyping of polymorphisms -1260/ C>A in CYP27B1, Lys198Asn in EDN1 and Ile105Val in GSTP1 by means of polymerase chain reaction (PCR) with allele-specific oligonucleotides (ASO-PCR) was performed. Primers used in ASO-PCR are listed in Table 1. Primers were designed using the Primer3 software (http://frodo.wi.mit.edu/primer3/input.htm). They were designed to amplify the target PCR products at similar temperature conditions, and two multiplex reactions were performed to genotype each individual at all three polymorphisms. The multiplex reaction contained three pairs of primers, each pair consisting of one common primer and one of the two allele-specific primers for each polymorphism. The final $10-\mu \mathrm{L}$ multiplex reaction mix contained: $1 \mathrm{X} \mathrm{GoTaq}{ }^{\circledR}$ Green reaction buffer (Promega, Madison, WI, USA), $0.2 \mathrm{mM}$ dNTP, $2 \mathrm{mM} \mathrm{MgCl}_{2}, 0.5$ unit GoTaq ${ }^{\circledR}$ polymerase, bidestilated $\mathrm{H}_{2} \mathrm{O}$ and 500 ng DNA. Primers CYP27B1FA, CYP27B1FC and CYP27B1R were used at 300 nanomolar concentrations and other primers were used at 200 nanomolar concentrations.

Two reaction mixes were used with similar constituents but differing in the added allele-specific primers: the first consisted of CYP27B1FA, EDN1RT and GSTP1RA primers and the second of CYP27B1FC, EDN1RG and GSTP1RG primers.

PCR were performed using Biometra TGradient Thermocycler (Biometra, Göttingen, Germany). For the first reaction mix, the conditions for PCR were as follows: initial denaturation at $95^{\circ} \mathrm{C}$ for $2 \mathrm{~min}$, then 35 cycles of denaturation at $95^{\circ} \mathrm{C}$, annealing at $65^{\circ} \mathrm{C}$ and elongation at $72^{\circ} \mathrm{C}$, and after the cycling final elongation at $72^{\circ} \mathrm{C}$ for $5 \mathrm{~min}$. Initial non-specific bands were resolved using a touchdown PCR protocol with annealing temperatures decreasing from $72^{\circ}$ to $65^{\circ} \mathrm{C}$ by a decrement of $0.7^{\circ} \mathrm{C}$ in each of the first 10 cycles, and subsequently, the annealing temperature was set at $65^{\circ} \mathrm{C}$ for the next 25 cycles. For the second reaction mix, the conditions for PCR were: initial denaturation at $95^{\circ} \mathrm{C}$ for $2 \mathrm{~min}$, then 35 cycles of denaturation at $95^{\circ} \mathrm{C}$, annealing at $61^{\circ}$ and elongation at $72^{\circ} \mathrm{C}$, and after the cycling final elongation at $72^{\circ} \mathrm{C}$ for $5 \mathrm{~min}$. The touchdown PCR protocol was also used for the second reaction mix, decreasing the annealing temperature from $67^{\circ}$ to $61^{\circ} \mathrm{C}$ in the first 10 cycles, after which the annealing temperature was set at $61^{\circ} \mathrm{C}$ for the next 25 cycles.

The presence of an amplified band indicated the presence of a certain SNP variant - the presence of 727, 394 and 168 bp bands signified the presence of a selected GSTP1/ 
Ile105Val, EDN1/ Lys198Asn and CYP27B1/ -1260/ C>A allelic variant, respectively. The products of PCR were visualized by electrophoresis on $2 \%$ agarose gels, stained with SYBR Safe ${ }^{\mathrm{TM}}$ fluorescent dye (Invitrogen, California, USA).

\begin{tabular}{llc}
\multicolumn{2}{c}{ Table 1. Sequences of primers used in the multiplex polymerase chain reactions with allele-specific aligonucleotides. } \\
\hline CYP27B1 (-1260) Reverse & CYP27B1R & 5' CGTATGCCTGTAGTGCCTTGAGA 3' \\
CYP27B1 (-1260/ A>G) Forward A-specific & CYP27B1FA & 5' GCAGAGAGGTAAACTGTGGGAGATTA 3' \\
CYP27B1 (-1260) Forward C-specific & CYP27B1FC & 5' GCAGAGAGGTAAACTGTGGGAGATTC 3' \\
EDN1 (Lys198Asn) Forward & EDN1F & 5' GATGAAGGTTGGGATGGGGC 3' \\
EDN1 (Lys198Asn) Reverse G-specific & EDN1RG & 5' CACATAACGCTCTCTGGAGGGC 3' \\
EDN1 (Lys198Asn) Reverse T-specific & EDN1RA & 5' CACATAACGCTCTCTGGAGGGA 3' \\
GSTP1 (Ile105Val) Forward & GSTP1F & 5' AGGAGGTGGTGACCGTGGAGA 3' \\
GSTP1 (Ile105Val) Reverse A-specific & GSTP1RA & 5' CACATAGTTGGTGTAGATGAGGGAGAT 3' \\
GSTP1 (Ile105Val) Reverse G-specific & GSTP1RG & 5' CACATAGTTGGTGTAGATGAGGGAGAC 3' \\
\hline
\end{tabular}

The G variant at EDN1/ Lys198Asn polymorphism codes for lysine amino acid and the T variant codes for asparagine. The A variant at the GSTP1/ Ile105Val polymorphism codes for isoleucine and the G variant codes for valine.

\section{Statistical analyses}

We analyzed the significance of the association using the chi-square test $\left(\chi^{2}\right)$. Odds ratios (OR) and their respective $95 \%$ confidence intervals $(\mathrm{CI})$ were also calculated to compare the allelic and genotype distributions in patients and control subjects. The analyses were performed using SPSS package (SPSS 14.0, SPSS Inc., Chicago, IL, USA) and the MedCalc 8.2 statistical software. Test of statistical power was performed using the PS Power and Sample Size Calculations program, version 2.1.30, available at http://biostat.mc.vanderbilt.edu/twiki/bin/view/Main/ PowerSampleSize (Dupont and Plummer Jr., 1997). Associations were regarded as significant when they reached a $P$ value of equal to or less than 0.05 .

\section{RESULTS}

We used data from four proteomic studies (Magi et al., 2002; Sabounchi-Schutt et al., 2003, 2004; Kriegova et al., 2006), three transcriptomic studies (Thonhofer et al., 2002; Rutherford et al., 2001, 2004) and two whole-genome linkage studies (Schurmann et al., 2001; Iannuzzi et al., 2005). We assembled a list of 35 potential candidate genes that were pathophysiologically related to sarcoidosis in several different pathways. Of these candidate genes, 19 were involved in the regulation of immune response, five in the regulation of cellular redox balance and protection from oxidative stress, five were implicated in apoptosis pathways, four were related to maintaining structures and regulating intercellular adhesions, three were associated with calcium metabolism, and two were implicated in carcinogenesis (some genes were involved in several pathways). Seven of the selected genes were located on chromosome 6 , five on chromosome 1 , four on chromosome 9 , three on chromosomes 3 and 4, and other genes were located separately on other chromosomes. Among all the candidates, three were selected for investigating the association with sarcoidosis.

There was no significant association of allelic variants of polymorphisms -1260 / C $>\mathrm{A}$ in CYP27B1 $(\mathrm{P}=0.70, \mathrm{OR}=1.07,95 \% \mathrm{CI}=0.80-1.44)$, Lys198Asn in EDN1 $(\mathrm{P}=1.00, \mathrm{OR}=$ $0.99,95 \% \mathrm{CI}=0.71-1.38)$ and Ile105Val in $\operatorname{GSTP} 1(\mathrm{P}=0.63, \mathrm{OR}=0.96,95 \% \mathrm{CI}=0.71-1.30)$ with susceptibility to sarcoidosis. 
Association with this disease also failed to reach significance with genotypes CC at -1260/ $\mathrm{C}>\mathrm{A}$ polymorphism in CYP27B1 $(\mathrm{P}=0.68, \mathrm{OR}=1.10,95 \% \mathrm{CI}=0.75-1.61), \mathrm{GG}$ at Lys198Asn polymorphism in $E D N 1(\mathrm{P}=1.00, \mathrm{OR}=0.97,95 \% \mathrm{CI}=0.65-1.44)$ and $\mathrm{AA}$ at Ile105Val polymorphism in GSTP1 $(\mathrm{P}=0.53, \mathrm{OR}=0.87,95 \% \mathrm{CI}=0.60-1.27)$, as shown in Table 2.

Table 2. Allele and genotype frequencies of CYP27B1/ -1260C >A polymorphism, EDN1/ Lys198Asn polymorphism and of GSTP1/ Ile150Val polymorphism in sarcoidosis patients and controls.

\begin{tabular}{|c|c|c|c|c|c|}
\hline Allele/Genotype & & Sarcoidosis N (\%) & Controls N (\%) & OR $(95 \% \mathrm{CI})$ & $\mathrm{P}$ \\
\hline \multicolumn{6}{|l|}{ CYP27B1 } \\
\hline \multirow[t]{3}{*}{ Allele } & $\mathrm{C}$ & $250(71.0)$ & $370(69.5)$ & $1.07(0.80$ to 1.44$)$ & 0.70 \\
\hline & A & $102(29.0)$ & $162(30.5)$ & & \\
\hline & Total & $352(100.0)$ & $532(100.0)$ & & \\
\hline \multirow[t]{4}{*}{ Genotype } & $\mathrm{CC}$ & $89(50.6)$ & $128(48.1)$ & $\mathrm{CC} v s \mathrm{CA}+\mathrm{AA}$ & 0.68 \\
\hline & $\mathrm{CA}$ & $72(40.9)$ & $114(42.9)$ & $1.10(0.75$ to 1.61$)$ & \\
\hline & AA & $15(8.5)$ & $24(9.0)$ & & \\
\hline & Total & $176(100.0)$ & $266(100.0)$ & & \\
\hline \multicolumn{6}{|l|}{ EDN1 } \\
\hline \multirow[t]{3}{*}{ Allele } & G & $270(78.9)$ & $426(79.2)$ & $0.99(0.71$ to 1.38$)$ & 1.00 \\
\hline & $\mathrm{T}$ & $72(21.1)$ & $112(20.8)$ & & \\
\hline & Total & $342(100.0)$ & $538(100.0)$ & & \\
\hline \multirow[t]{4}{*}{ Genotype } & GG & $105(61.4)$ & $167(62.1)$ & $\mathrm{GG} v s \mathrm{GT}+\mathrm{TT}$ & 1.00 \\
\hline & GT & $60(35.1)$ & $92(34.2)$ & $0.97(0.65$ to 1.44$)$ & \\
\hline & TT & $6(3.5)$ & $10(3.7)$ & & \\
\hline & Total & $171(100.0)$ & $269(100.0)$ & & \\
\hline \multicolumn{6}{|l|}{ GSTP1 } \\
\hline \multirow[t]{3}{*}{ Allele } & G & $97(27.2)$ & $144(26.5)$ & $0.96(0.71$ to 1.30$)$ & 0.63 \\
\hline & A & $259(72.8)$ & $400(73.5)$ & & \\
\hline & Total & $356(100.0)$ & $544(100.0)$ & & \\
\hline \multirow[t]{4}{*}{ Genotype } & GG & $9(5.1)$ & $19(7.0)$ & $\mathrm{AA} v s \mathrm{GG}+\mathrm{GA}$ & 0.53 \\
\hline & GA & $79(44.4)$ & $106(39.0)$ & $0.87(0.60$ to 1.27$)$ & \\
\hline & $\mathrm{AA}$ & $90(50.6)$ & $147(54.0)$ & & \\
\hline & Total & $178(100.0)$ & $272(100.0)$ & & \\
\hline
\end{tabular}

The G variant at EDN1/ Lys198Asn polymorphism codes for lysine amino acid and the T variant codes for asparagine. The A variant at the GSTP1/ Ile105Val polymorphism codes for isoleucine and the G variant codes for valine.

The calculation of statistical power showed that in the analyses of the three polymorphisms the power should be $80 \%$ to detect a 1.73 -fold increase in the frequencies of risk genotypes at $-1260 \mathrm{C}>\mathrm{A} / \mathrm{CYP} 27 \mathrm{~B} 1$ polymorphism, assuming that the percentage of risk genotypes in controls was $48.1 \%$. The power should be $80 \%$ to detect a 1.82 -fold increase in the frequencies of risk genotypes at EDN1/ Lys198Asn polymorphism, assuming that the percentage of risk genotypes in controls was $62.1 \%$. For the GSTP1/ Ile105Val polymorphism, the power should be $80 \%$ to detect a 1.75 -fold increase in the frequencies of risk genotypes, assuming that the percentage of risk genotypes in controls was $54.0 \%$.

There was no significant deviation from the frequencies of genotypes predicted by Hardy-Weinberg equilibrium $(\mathrm{P}<0.05)$.

\section{DISCUSSION}

We performed a comprehensive literature search for sarcoidosis candidate genes; this revealed a number of possible genes and their respective variants that could be implicated in 
the etiopathogenesis of sarcoidosis, but had not been considered in previous genetic association studies. These associations had not been considered previously because the genes' functions do not have a straightforward relationship with pathogenetic processes in sarcoidosis. A systematic approach enabled us to accept these genes as possible candidates on the basis of linkage, transcriptomic, proteomic, and recent functional studies (Schurmann et al., 2001; Sabounchi-Schutt et al., 2003; Rutherford et al., 2004). This approach has revealed candidate genes, whose ancillary functions have only recently been implicated in the pathogenesis of several lung diseases, and whose altered transcriptomic or proteomic profile as well as functional changes could contribute to the pathogenesis of sarcoidosis. We chose to study polymorphisms in the genes that play a role in blood pressure regulation $(E D N 1)$, vitamin D metabolism (CYP27B1) and detoxification reactions (GSTP1), all of which have only recently been assigned direct and indirect immunomodulatory roles.

CYP27B1 codes for a cytochrome enzyme that catalyses $1 \alpha$-hydroxylation of vitamin D to produce its more active form (Monkawa et al., 1997). Vitamin D and its metabolism have been implicated in the pathogenesis of sarcoidosis, which is characterized by increased concentration of serum calcium and increased renal excretion of calcium (Sharma, 1996). Vitamin $\mathrm{D}_{3}-1 \alpha$-hydroxylase was implicated in the pathogenesis of sarcoidosis after it was hypothesized that hypercalcemic and hypercalciuric manifestations of sarcoidosis could be due to extrarenal overproduction of an active form of vitamin D hormone $\left(1,25-[\mathrm{OH}]_{2}-\mathrm{D}_{3}\right)$ from macrophages in granulomatous lesions (Adams et al., 1983). This was later substantiated by analyses of vitamin D conversion in lymph node samples and alveolar macrophages obtained from patients with sarcoidosis (Adams et al., 1983), and further confirmed by expression studies that showed that macrophages in sarcoid lesions expressed CYP27B1 (Zehnder et al., 2001). The levels of CYP27B1 mRNA could also be paralleled with disease activity in a study by Inui et al. (2001). The cause of this induction in macrophage cells has been shown to be interferon- $\gamma$ (IFN- $\gamma$ ), which could stimulate the expression of CYP27B1 in macrophages from sarcoid lesions by itself, in contrast to macrophages isolated from healthy individuals (Vidal et al., 2002).

The role of vitamin $\mathrm{D}$ in the regulation of the immune response has also been recognized; alterations in vitamin D function are related to several autoimmune diseases, such as inflammatory bowel diseases, autoimmune diabetes mellitus, autoimmune thyroiditis, and others (Cantorna et al., 2004). The active form of vitamin D $\left(1,25-[\mathrm{OH}]_{2}-\mathrm{D}_{3}\right.$ or calcitriol) has been reported to induce the transformation of circulating monocytes into epithelioid cells and multinucleated giant cells, which are characteristic of granulomatous lesions in sarcoidosis (Ohta et al., 1986). For these reasons, polymorphisms in genes coding for proteins involved in vitamin $\mathrm{D}$ function have been previously studied in sarcoidosis, but the existing association studies of polymorphisms in vitamin $\mathrm{D}$ receptor gene $(V D R)$ have given conflicting results (Niimi et al., 2000; Rybicki et al., 2004), although a more recent familial-based association study by Rybicki et al. (2004) renewed the interest in the role of VDR gene. Since macrophages also express vitamin $\mathrm{D}$ receptors, dysregulation of autocrine and paracrine vitamin D-regulated loop could be a cause of persistent inflammation in sarcoidosis. Taking all this data into consideration, an intricate association between extrarenal vitamin D metabolism and inflammation in sarcoidosis may be established.

Mutations within CYP27B1, which impair vitamin $\mathrm{D}_{3}-1 \alpha$-hydroxylase activity and cause vitamin D-dependent rickets, have been described previously (Miller and Portale, 2000). An SNP at position -1260 in the gene promoter region has also been described; it causes a 
change in the nucleotide $\mathrm{C}$ to A. The SNP is located in the AP-2 transcription factor binding motif and in a region that has been shown to have an inhibitory influence on the transcription of the CYP27B1 gene (Kong et al., 1999). CYP27B1 has also been shown to be under the inhibitory influence of the nuclear factor-kappaB (NF- $\kappa B)$ transcription factor, suggesting its importance in an inflammatory response (Ebert et al., 2004). The $\mathrm{C}$ variant of this SNP has been associated with Hashimoto's thyroiditis, Addison's disease, Graves' disease, and autoimmune diabetes mellitus (Lopez et al., 2004). The functional role of this polymorphism has not yet been determined, but there is a study that has reported an increased number of -1260/ CA heterozygotes in patients with normal production of calcitriol, possibly indicating its functional significance (Ramos-Lopez et al., 2007). We failed to show an association of sarcoidosis with the $\mathrm{C}$ variant and predicted risk genotype $\mathrm{CC}$ at $C Y P 27 B 1 /-1260 \mathrm{C}>\mathrm{A}$ polymorphism in Slovenian patients with sarcoidosis. The association with this disease failed to reach significance with the predicted high-risk genotype $\mathrm{CC}$ at -1260 / C $>$ A polymorphism in the CYP27B1 gene $(\mathrm{P}=0.68, \mathrm{OR}=1.10,95 \% \mathrm{CI}=0.75-1.61)$.

Another investigated gene, EDN1, coding for vasoactive factor ET-1, was also considered, as its levels have been consistently found elevated in patients with sarcoidosis in various studies (Reichenberger et al., 2001; Terashita et al., 2006). ET-1 is a powerful vasoconstricting peptide with recently recognized immunoregulatory properties, acting as an inflammatory cytokine, stimulating macrophages to secrete pro-inflammatory cytokines (Cui et al., 2001). ET-1 has generally been implicated in the pathogenesis of lung diseases characterized by fibrosis, including sarcoidosis, on the basis of its proliferative and mitogenic effects on vascular smooth muscle cells and fibroblasts in vitro and its pattern of expression in lung endothelial and epithelial cells as well as alveolar macrophages (Reichenberger et al., 2001; Terashita et al., 2006). Increased concentrations of ET-1 in bronchoalveolar lavage fluid have been measured in patients with fibrotic lung diseases; Terashita et al. (2006), Reichenberger et al. (2001) and $\mathrm{Li}$ et al. (1999) have demonstrated increased ET-1 concentration in bronchoalveolar lavage fluid samples from patients with sarcoidosis. It has been demonstrated that ET-1 concentrations rise and fall in accordance with disease activity and remission, respectively (Letizia et al., 2001). Increased urinary excretion of ET-1 in sarcoidosis has also been described (Sofia et al., 1995). The gene coding for ET-1 is polymorphic: an SNP causing amino acid change from lysine to asparagine at position 198 in coding region has been associated with hypertension, HDL cholesterol levels and IgA nephropathy progression, suggesting its functional significance (Jin et al., 2003). In our study, we did not observe any significant association of allelic variants at $E D N 1 /$ Lys198Asn polymorphism with sarcoidosis susceptibility. Also, we failed to show a significant association of the predicted low-risk genotype GG at Lys198Asn polymorphism in the $E D N 1$ gene $(\mathrm{P}=1.00, \mathrm{OR}=0.97,95 \% \mathrm{CI}=0.65-1.44)$ and sarcoidosis.

GSTP1 gene codes for a member of the glutathione S-transferase (GST) family with established roles in the pathogenesis of cancer, asthma, rheumatoid arthritis, and other inflammatory diseases (Fryer et al., 2000). Sarcoidosis is characterized by a significantly decreased amount of GSTP1 in bronchoalveolar lavage fluid obtained from patients (Sabounchi-Schutt et al., 2003).

GSTs are a family of enzymes that catalyze conjugation reactions of various substances, including various ROS, with glutathione, therefore being essential for the protection of cells from oxidative stress caused by ROS. The effect of oxidative stress on persistence of inflammatory processes has been implicated as a possible factor in the pathogenesis of sarcoidosis, which is also characterized by increased extent of oxidative damage of proteins 
and increased levels of oxidative stress markers in bronchoalveolar lavage fluid samples (Rahman, 2003). In addition to their damaging effect on tissues, ROS have been attributed immunomodulatory functions in the lung, initiating immune responses through the activation of transcription factors (NF- $\mathrm{KB})$ and activator protein-1 (AP-1), as well as affecting signal transduction and gene expression of pro-inflammatory factors. Epithelial lung cells and macrophages have been shown to exhibit increased expression of $I L-1$ and $T N F-\alpha$ after exposing them to ROS in vitro (Rahman, 2003). The imbalance between actions of proand anti-inflammatory mediators in sarcoidosis could therefore be potentiated by oxidative stress, as a consequence of diminished activity of GSTP1 enzyme. An SNP has been found in the GSTP1 gene that confers amino acid changes in its protein product from isoleucine to valine at position 105 (Ile105Val), causing a 30\% diminished activity of GSTP1 enzyme (Ali-Osman et al., 1997). The presence of this variant in GSTP1 could reduce the activity of cellular antioxidant mechanisms, potentiating oxidative damage and increasing amounts of ROS, thereby causing persistent inflammation that is present in sarcoidosis. The results of genotyping at this polymorphism in our study did not show that variants of this polymorphism were at significant risk for susceptibility to sarcoidosis.

Also, the association with this disease failed to reach significance with the predicted low-risk genotype AA at Ile105Val polymorphism in the GSTP1 gene $(\mathrm{P}=0.53$, OR $=0.87,95 \% \mathrm{CI}=0.60-1.27$ ).

Our results have failed to show significant differences between the sarcoidosis patients and the controls concerning the EDN1, CYP27B1 and GSTP1 genetic polymorphisms. However, we cannot exclude a possible association of these candidate genes with sarcoidosis. The lack of significant associations could be due to relatively small study sample, considering that we achieved a power of $80 \%$ to detect a somewhat large 1.7 -fold increased frequency of risk genotypes in patients with sarcoidosis. This has limited our ability to reach significant results for the variants with minor effects on disease susceptibility, which are probably important in genetic susceptibility to sarcoidosis, and/or clinical presentation of the disease. Complex gene-gene and gene-environment interactions should also be considered in sarcoidosis etiology. Only one of several possible variants in the region of each gene has been studied, therefore, other variants in genes, alone or through haplotype combinations should be investigated for possible associations with sarcoidosis.

\section{ACKNOWLEDGMENTS}

Research supported by Slovenian Research Agency grant \#P3-0326.

\section{REFERENCES}

Adams JS, Sharma OP, Gacad MA and Singer FR (1983). Metabolism of 25-hydroxyvitamin D3 by cultured pulmonary alveolar macrophages in sarcoidosis. J. Clin. Invest. 72: 1856-1860.

Ali-Osman F, Akande O, Antoun G, Mao JX, et al. (1997). Molecular cloning, characterization, and expression in Escherichia coli of full-length cDNAs of three human glutathione S-transferase Pi gene variants. Evidence for differential catalytic activity of the encoded proteins. J. Biol. Chem. 272: 10004-10012.

Baughman RP, Lower EE and du Bois RM (2003). Sarcoidosis. Lancet 361: 1111-1118.

Cantorna MT, Zhu Y, Froicu M and Wittke A (2004). Vitamin D status, 1,25-dihydroxyvitamin D3, and the immune system. Am. J. Clin. Nutr. 80: 1717S-1720S.

Cui P, Tani K, Kitamura H, Okumura Y, et al. (2001). A novel bioactive 31-amino acid endothelin-1 is a potent chemotactic 
peptide for human neutrophils and monocytes. J. Leukoc. Biol. 70: 306-312.

Dupont WD and Plummer WD Jr (1997). PS power and sample size program available for free on the internet. Control Clin. Trials 18: 274.

Ebert R, Jovanovic M, Ulmer M, Schneider D, et al. (2004). Down-regulation by nuclear factor kappaB of human 25-hydroxyvitamin D3 1alpha-hydroxylase promoter. Mol. Endocrinol. 18: 2440-2450.

Fryer AA, Bianco A, Hepple M, Jones PW, et al. (2000). Polymorphism at the glutathione S-transferase GSTP1 locus. A new marker for bronchial hyperresponsiveness and asthma. Am. J. Respir. Crit. Care Med. 161: 1437-1442.

Hunninghake GW, Costabel U, Ando M, Baughman R, et al. (1999). ATS/ERS/WASOG statement on sarcoidosis. American Thoracic Society/European Respiratory Society/World Association of Sarcoidosis and other Granulomatous Disorders. Sarcoidosis Vasc. Diffuse Lung Dis. 16: 149-173.

Iannuzzi MC, Iyengar SK, Gray-McGuire C, Elston RC, et al. (2005). Genome-wide search for sarcoidosis susceptibility genes in African Americans. Genes Immun. 6: 509-518.

Inui N, Murayama A, Sasaki S, Suda T, et al. (2001). Correlation between 25-hydroxyvitamin D3 1 alpha-hydroxylase gene expression in alveolar macrophages and the activity of sarcoidosis. Am. J. Med. 110: 687-693.

Jin JJ, Nakura J, Wu Z, Yamamoto M, et al. (2003). Association of endothelin-1 gene variant with hypertension. Hypertension 41: 163-167.

Kong XF, Zhu XH, Pei YL, Jackson DM, et al. (1999). Molecular cloning, characterization, and promoter analysis of the human 25-hydroxyvitamin D3-1alpha-hydroxylase gene. Proc. Natl. Acad. Sci. U. S. A. 96: 6988-6993.

Kriegova E, Melle C, Kolek V, Hutyrova B, et al. (2006). Protein profiles of bronchoalveolar lavage fluid from patients with pulmonary sarcoidosis. Am. J. Respir. Crit. Care Med. 173: 1145-1154.

Letizia C, Danese A, Reale MG, Caliumi C, et al. (2001). Plasma levels of endothelin-1 increase in patients with sarcoidosis and fall after disease remission. Panminerva Med. 43: 257-261.

Li Z, Xia S and Hou X (1999). [Changes of the activities of ET-1 in the serum and BALF of ILD patients and their clinical significance]. Zhonghua Jiehe He Huxi Zazhi 22: 411-413.

Lopez ER, Zwermann O, Segni M, Meyer G, et al. (2004). A promoter polymorphism of the CYP27B1 gene is associated with Addison's disease, Hashimoto's thyroiditis, Graves' disease and type 1 diabetes mellitus in Germans. Eur. $J$. Endocrinol. 151: 193-197.

Magi B, Bini L, Perari MG, Fossi A, et al. (2002). Bronchoalveolar lavage fluid protein composition in patients with sarcoidosis and idiopathic pulmonary fibrosis: a two-dimensional electrophoretic study. Electrophoresis 23: 3434-3444.

Miller WL and Portale AA (2000). Vitamin D 1 alpha-hydroxylase. Trends Endocrinol. Metab. 11: 315-319.

Monkawa T, Yoshida T, Wakino S, Shinki T, et al. (1997). Molecular cloning of cDNA and genomic DNA for human 25-hydroxyvitamin D3 1 alpha-hydroxylase. Biochem. Biophys. Res. Commun. 239: 527-533.

Montano MM, Deng H, Liu M, Sun X, et al. (2004). Transcriptional regulation by the estrogen receptor of antioxidative stress enzymes and its functional implications. Oncogene 23: 2442-2453.

Niimi T, Tomita H, Sato S, Akita K, et al. (2000). Vitamin D receptor gene polymorphism and calcium metabolism in sarcoidosis patients. Sarcoidosis Vasc. Diffuse Lung Dis. 17: 266-269.

Ohta M, Okabe T, Ozawa K, Urabe A, et al. (1986). In vitro formation of macrophage-epithelioid cells and multinucleated giant cells by 1 alpha,25-dihydroxyvitamin D3 from human circulating monocytes. Ann. N. Y. Acad. Sci. 465: 211-220.

Rahman I (2003). Oxidative stress, chromatin remodeling and gene transcription in inflammation and chronic lung diseases. J. Biochem. Mol. Biol. 36: 95-109.

Ramos-Lopez E, Brück P, Jansen T, Pfeilschifter JM, et al. (2007). CYP2R1-, CYP27B1- and CYP24-mRNA expression in German type 1 diabetes patients. J. Steroid Biochem. Mol. Biol. 103: 807-810.

Reichenberger F, Schauer J, Kellner K, Sack U, et al. (2001). Different expression of endothelin in the bronchoalveolar lavage in patients with pulmonary diseases. Lung 179: 163-174.

Rutherford RM, Kehren J, Staedtler F, Chibout SD, et al. (2001). Functional genomics in sarcoidosis - reduced or increased apoptosis? Swiss Med. Wkly. 131: 459-470.

Rutherford RM, Staedtler F, Kehren J, Chibout SD, et al. (2004). Functional genomics and prognosis in sarcoidosis - the critical role of antigen presentation. Sarcoidosis Vasc. Diffuse Lung Dis. 21: 10-18.

Rybicki BA, Maliarik MJ, Poisson LM and Iannuzzi MC (2004). Sarcoidosis and granuloma genes: a family-based study in African-Americans. Eur. Respir. J. 24: 251-257.

Sabounchi-Schutt F, Astrom J, Hellman U, Eklund A, et al. (2003). Changes in bronchoalveolar lavage fluid proteins in sarcoidosis: a proteomics approach. Eur. Respir. J. 21: 414-420.

Sabounchi-Schutt F, Mikko M, Eklund A, Grunewald J, et al. (2004). Serum protein pattern in sarcoidosis analysed by a proteomics approach. Sarcoidosis Vasc. Diffuse Lung Dis. 21: 182-190.

Schurmann M, Reichel P, Muller-Myhsok B, Schlaak M, et al. (2001). Results from a genome-wide search for predisposing genes in sarcoidosis. Am. J. Respir. Crit. Care Med. 164: 840-846. 
Sharma OP (1996). Vitamin D, calcium, and sarcoidosis. Chest 109: 535-539.

Sofia M, Mormile M, Faraone S, Alifano M, et al. (1995). Endothelin-1 excretion in urine in active pulmonary sarcoidosis and in other interstitial lung diseases. Sarcoidosis 12: 118-123.

Terashita K, Kato S, Sata M, Inoue S, et al. (2006). Increased endothelin-1 levels of BAL fluid in patients with pulmonary sarcoidosis. Respirology 11: 145-151.

Thonhofer R, Maercker C and Popper HH (2002). Expression of sarcoidosis related genes in lung lavage cells. Sarcoidosis Vasc. Diffuse Lung Dis. 19: 59-65.

Vidal M, Ramana CV and Dusso AS (2002). Stat1-vitamin D receptor interactions antagonize 1,25-dihydroxyvitamin D transcriptional activity and enhance stat1-mediated transcription. Mol. Cell Biol. 22: 2777-2787.

Zehnder D, Bland R, Williams MC, McNinch RW, et al. (2001). Extrarenal expression of 25-hydroxyvitamin d(3)-1 alpha-hydroxylase. J. Clin. Endocrinol. Metab. 86: 888-894. 